\title{
Entrevista motivacional y actitudes ante el cambio en pacientes con un trastorno del comportamiento alimentario
}

\author{
Beato Fernández, L.*; Rodríguez Cano, T.** \\ * Psiquiatra. Unidad de Trastornos Alimentarios. Servicio de Psiquiatría. Complejo Hospitalario de Ciudad Real. \\ * Psiquiatra. Servicio de Psiquiatría. Complejo Hospitalario de Ciudad Real.

\section{RESUMEN}

Presentamos aquí los resultados preliminares de un estudio en el que, basándonos en los principios de la técnica de entrevista motivacional y siguiendo el modelo transteórico del cambio propuesto por Prochaska y DiClemente, hemos diseñado un cuestionario específico para valorar las Actitudes ante el Cambio en pacientes con Trastornos Alimentarios (ACTA). Posteriormente hemos correlacionado las distintas subescalas de este, con las puntuaciones a los seis meses en diversos cuestionarios que valoran psicopatología alimentaria. Encontramos que altas puntuaciones en la escala de mantenimiento eran un factor protector de la psicopatología alimentaria, medida con el EAT-40 a los seis meses. Las altas puntuaciones en la fase de decisión eran predictoras del grado de insatisfacción con la imagen corporal, así como las puntuaciones en la escala de contemplación también predecían la sintomatología bulímica, medida con la subescala de síntomas del BITE a los seis meses.

Palabras clave: Trastornos del Comportamiento Alimentario, Anorexia nerviosa, Bulimia nerviosa, Terapia Motivacional, Evaluación del cambio.

\section{SUMMARY}

We present here the preliminary results of a research work in which, based on the principles of motivational interviewing and following the transtheoretical model of change, proposed by Prochaska and DiClemente, we designed a questionnaire to assess the attitudes towards change in eating disordered patients (ACTA.).The correlation between the ACTA subscales scores and several questionnaires, that evaluate eating disorder psychopathology, were analysed. We found that high scores on maintenance subscale was a protective factor of eating psychopathology, measured by the EAT-40 six months later. High scores on decision step was predictive for the level of dissatisfaction with own body image In addition, scores on the contemplation scale predicted bulimic symptoms measured with the BITE symptoms subscale six months later.

Key words: Eating Disorders, Anorexia nervosa, Bulimia nervosa, Motivational Enhancement Therapy, Change assessment.

pabilización por las repercusiones negativas de su conducta, o incluso amenazas por parte de figuras de autoridad. Respecto al tratamiento también encontramos similitudes entre ambos grupos de trastornos: no disponemos de fármacos suficientemente eficaces y, para la modificación de las conductas patológicas, es necesaria la colaboración del paciente. El pronóstico también está influido por el tiempo de evolución del problema, de manera que existe urgencia en conseguir la remisión de estos patrones de conducta anómalos.

La técnica de entrevista motivacional ha mostrado eficacia en el abordaje de los pacientes con conductas adictivas, por lo tanto, es de suponer que su adapta- 
ción a los trastornos de la conducta alimentaria podría ser también eficaz en el enfoque psicoterapéutico de algunos de nuestros pacientes. Conocemos estudios llevados a cabo con este enfoque en los pacientes con un trastorno del comportamiento alimentario. Diversas investigaciones realizadas por el grupo de la Unidad de Trastornos Alimentarios del 'Maudsley Hospital' de Londres y, últimamente replicadas en un trabajo del Departamento de Psiquiatría de la Universidad de Toronto, concluyen que el modelo transteórico de Prochaska y DiClemente (2) es un enfoque útil en el abordaje psicoterapéutico de estos pacientes (3-5). Originariamente aplicado a una población de fumadores, el objetivo es entender y promover el cambio examinando el estadío en que se encuentra el individuo. Las fases descritas se pueden definir como: Precontemplación, antes de que el individuo reconozca que tiene un problema; contemplación, sabe que tiene un problema pero no siente la necesidad de resolverlo; decisión, el individuo ha decidido cambiar en un futuro pero aun no ha iniciado el cambio; acción, el individuo comienza a trabajar en su conducta y necesita ayuda para alcanzar el cambio deseado; mantenimiento, se alcanza el cambio; la recaída es también una fase dentro de este proceso de cambio.

Las características de nuestro entorno de trabajo han favorecido la implantación por nuestro equipo de esta técnica. Habitualmente recibimos pacientes adultos con un promedio de 5 años de evolución del trastorno y en quienes se han ensayado dos o más tratamientos previos. El apoyo familiar para el control de conductas patológicas, o para mantenerles en el programa de tratamiento ambulatorio, resulta habitualmente deficitario. En esta situación, es imprescindible contar con la colaboración del paciente para iniciar y mantener el programa terapéutico. Los pacientes con un trastorno del comportamiento alimentario se muestran ambivalentes con respecto al tratamiento. Con frecuencia acuden a consulta conducidos por sus familiares o como consecuencia de algún síntoma físico que les ha obligado a solicitar atención en los servicios de urgencias. La resistencia ante las indicaciones terapéuticas, así como los abandonos, son fenómenos frecuentemente observados entre los clínicos que atendemos estos pacientes y, según este modelo teórico, los fracasos en el tratamiento suelen ser secundarios a que las indicaciones no son apropiadas al estado de cambio en el que está el paciente (6).

Presentamos los resultados preliminares de un trabajo que estamos Ilevando a cabo en la Unidad de Trastornos Alimentarios del Hospital de Ciudad Real. Integrando el modelo del cambio con las estrategias de la entrevista motivacional, hemos elaborado un cuestionario que evalúa la actitud frente al cambio en pacientes con TCA y lo hemos correlacionado con variables psicopatológicas en dos momentos, separa- dos por un intervalo de tiempo de seis meses. Se trata de un estudio piloto dentro de un proyecto que pretende identificar los pacientes que responderían mejor a determinadas estrategias psicoterapéuticas, prevenir y evitar abandonos, y acelerar el proceso de cambio en pacientes que presentan un trastorno psiquiátrico habitualmente de larga evolución y en muchos casos crónico.

\section{SUJETOS Y METODO}

\section{Descripción de la muestra}

La muestra estaba constituida por 37 pacientes del sexo femenino, seleccionadas aleatoriamente entre aquellas que acudían para recibir tratamiento a la Unidad de Trastornos Alimentarios del Complejo Hospitalario de Ciudad Real desde Agosto del 2000 a Agosto del 2001. Seis pacientes fueron eliminadas por no cumplimentar adecuadamente los cuestionarios, o por no completar los correspondientes a la primera y/o segunda entrevista. La edad media era de 22.16 \pm 5.59 años. Dieciocho (57.6\%) estaban solteras sin pareja, $10(32.2 \%)$ eran solteras pero tenían pareja y tres $(9.6 \%)$ estaban casadas. En cuanto al nivel de estudios, 13 pacientes (41.6\%) cursaban el bachiller o COU, 10 (32.2\%) una titulación media, 4 (12.8\%), cursaban estudios superiores, $3(9.6 \%)$ sólo habían estudiado la EGB o ESO y una paciente (3.2\%) no la había finalizado los estudios primarios.

Los diagnósticos fueron los siguientes: 11 (34.1\%) bulimia purgativa (BP), $7(21.7 \%)$ trastorno de conducta alimentaria no especificado (TCANE), 8 (24.8\%) anorexia restrictiva (AR), $3(9.3 \%)$ anorexia purgativa (AP) y $2(6.2 \%)$ bulimia no purgativa (BNP). El tiempo medio de evolución del trastorno era de $60.96 \pm 55.96$ meses (rango 5 a 240 meses) y el tiempo medio de inicio del tratamiento era de $16.03 \pm 28.47$ meses (rango 0 a 96 meses). Ninguna de las pacientes incluidas presentaban otras complicaciones físicas asociadas a su estado nutricional (obesidad, diabetes mellitus o alteraciones lipídicas) u otras alteraciones orgánicas que requirieran cuidados o tratamientos específicos. Fueron excluidas, asimismo, pacientes que presentaban sintomatología depresiva moderada o grave, síntomas de ansiedad intensos u otra psicopatología diferente a la de su trastorno alimentario. Ninguna de las pacientes estaba a la vez incluidas en otro programa de tratamiento diferente al llevado en nuestro centro. Todas las intervenciones terapéuticas eran registradas en su historia clínica, así como los progresos en su tratamiento

\section{Material y procedimiento}

Las pacientes eran evaluadas por un psiquiatra experimentado y el diagnóstico se realizó según los 
criterios de la DSM-IV para los trastornos de la conducta alimentaria (TCA). Como parte del examen, se les administró un cuestionario semiestructurado sobre variables sociodemográficas y clínicas.

La psicopatología alimentaria fue determinada con los siguientes cuestionarios:

-EAT-40. Cuestionario de screening para anorexia nerviosa, validado para población española por Castro, Toro, Salmero y Guimerá (7)

-BITE: Cuestionario autoaplicado que registra, a través de dos subescalas, síntomas y gravedad de la conducta bulímica. Existe una puntuación total que corresponde a la suma de ambas subescalas.

-BSQ. Este cuestionario pretende detectar el grado de insatisfacción que los pacientes sienten hacia su figura corporal. Validado en población española por R. Raich y cols (8).

Para evaluar la actitud frente al cambio, según el modelo de Prochaska y DiClemente, se elaboró un cuestionario autoaplicado tipo Likert, con cinco posibles respuestas, que inicialmente constaba de 73 preguntas, correspondientes a seis subescalas. Actualmente dicho cuestionario de "Actitudes frente al Cambio en los Trastornos Alimentarios" (A.C.T.A.), está siendo validado, mostrando una buena consistencia interna global (a de Cronbach $=0.8451$ ) y para las diferentes subescalas: precontemplación $(\alpha=0.7996)$, contemplación $(\alpha=0.7323)$, decisión ( $\alpha=0.7146$, después de eliminar las preguntas 5 y 14), acción ( $\alpha=0.8763)$, mantenimiento $(\alpha=0.9383)$ y recaída $(\alpha=0.8490)$.

\section{Intervención}

Todas las intervenciones terapéuticas se basaron en los principios de la técnica de entrevista motivacional: Enfoque del problema por parte del paciente, empezando a trabajar con el material que el paciente trae a la consulta; instauración en los padres de los principios de la entrevista motivacional; facilitar el análisis de pros y contras de sus hábitos y la aparición de ambivalencia; instauración de la iniciativa del trata- miento por parte del paciente; inicio de actitud de colaboración en las metas establecidas, incorporando técnicas cognitivo-conductuales.

\section{RESULTADOS}

\section{Frecuencia de pacientes en los distintos estadios del cambio.}

La tabla 1 muestra la proporción de pacientes que presentaban mayores puntuaciones en cada una de las fases de la motivación al inicio de estudio y seis meses después. La mayoría ( $n=14,44.8 \%)$ puntuaron más alto en la fase de acción, seguidas de: recaída $(n=7$, $22.4 \%)$, decisión $(n=6,19.2 \%)$, mantenimiento $(n=2$, $6.4 \%)$, contemplación $(n=1,3.2 \%)$, y precontemplación $(n=1,3.2 \%)$. A los seis meses de seguimiento, 13 $(41.6 \%)$ pacientes estaban en fase de acción, pero había aumentado el número de pacientes en fase de mantenimiento $(n=7,22.2 \%)$. El número de pacientes en recaída fue $8(25.6 \%)$. La tabla 2 muestra los cambios experimentados por los pacientes entre la primera y segunda entrevista respecto al estadio del cambio predominante, es decir, aquella subescala en la que las puntuaciones fueron mayores. Debido al tamaño de la muestra no se pudieron estudiar si las diferencias eran estadísticamente significativas.

Tabla 1. Frecuencia de pacientes en las distintas fase de motivación para el cambio.

\begin{tabular}{lrrrrc}
\hline FASE & \multicolumn{2}{c}{ AL INICIO } & \multicolumn{2}{c|}{ A LOS SEIS MESES } \\
\cline { 2 - 3 } & Fr & \% & & Fr & $\%$ \\
\cline { 2 - 3 } \cline { 5 - 6 } Precontemplación & 1 & 3,2 & & 1 & 3.2 \\
Contemplación & 1 & 3,2 & & 0 & 0 \\
Decisión & 6 & 19,2 & & 2 & 6,4 \\
Acción & 14 & 44,8 & & 13 & 41,6 \\
Mantenimiento & 2 & 6,4 & & 7 & 22,4 \\
Recaída & 7 & 22,4 & & 25,6 \\
\hline
\end{tabular}

Tabla 2. Número de pacientes que cambiaron de estadío entre la primera y segunda entrevista.

Tabla de contingencia estadío del cambio * estadío del cambio

\begin{tabular}{|c|c|c|c|c|c|c|c|}
\hline \multirow[b]{3}{*}{$\begin{array}{l}\text { Estadío } \\
\text { del } \\
\text { cambio }\end{array}$} & \multirow{4}{*}{$\begin{array}{l}\text { Precontemplación } \\
\text { Contemplación } \\
\text { Decisión } \\
\text { Acción } \\
\text { Mantenimiento } \\
\text { Recaída }\end{array}$} & \multicolumn{6}{|c|}{ Estadío del cambio a los seis meses } \\
\hline & & Precontemplación 2 & Contemplación 2 & Decisión 2 & Acción 2 & Mantenimiento 2 & Recaída 2 \\
\hline & & & & 2 & $\begin{array}{l}1 \\
2 \\
6 \\
1 \\
3\end{array}$ & $\begin{array}{l}1 \\
1 \\
4 \\
1\end{array}$ & $\begin{array}{l}1 \\
4\end{array}$ \\
\hline Total & & $\begin{array}{l}1 \\
1\end{array}$ & & 2 & 13 & 7 & $\begin{array}{l}3 \\
8\end{array}$ \\
\hline
\end{tabular}




\section{Correlación entre las distintas subescalas que} miden actitudes ante el cambio

Como muestra la tabla 3, la fase de precontemplación y contemplación se correlacionaba significativamente con decisión y recaída, e inversamente con mantenimiento. La fase de decisión también se correlacionaba con precontemplación, contemplación y recaída. La fase de recaída, igualmente, se correlaciona con precontemplación y contemplación e inversamente con mantenimiento. Por el contrario, las fases de mantenimiento y acción se correlacionan positivamente entre sí e inversamente con precontemplación y recaída.

La tabla cuatro muestra las correlaciones entre las puntuaciones de cada subescala al inicio y seis meses después. Se encontró una correlación moderada. En cuanto a las escalas que miden psicopatología alimentaria, también se encontró una correlación moderada en las puntuaciones obtenidas en el EAT-40, BITE y $\mathrm{BSO}$ al inicio del tratamiento y seis meses después.
Correlación entre las distintas subescalas que miden actitudes ante el cambio y las subescalas que miden psicopatología alimentaria

La fase de precontemplación se correlaciona significativamente con las puntuaciones en el EAT-40 y BSO ( $<$ < 0.01) (tabla 5) Las fases de contemplación, decisión y recaída se correlacionan significativamente con las puntuaciones en el EAT-40, BITE y BSO $(p<0.01)$. Por el contrario, las puntuaciones en la fase de acción mostraron una correlación inversa con las puntuaciones en el EAT-40 y las puntuaciones en la fase de mantenimiento también mostraron una correlación inversa con las puntuaciones en el EAT-40, BITE y BSO, es decir a mayores puntuaciones en la fase de acción y mantenimiento, menor psicopatología alimentaria. No se halló correlación entre las puntuaciones en las distintas subescalas del cuestionario de motivación y variables como duración del trastorno o tiempo transcurrido desde el inicio del tratamiento.

Tabla 3. Correlación entre las subescalas del Cuestionario de actitudes frente al cambio (ACTA)

Correlaciones

\begin{tabular}{|l|l|c|c|c|c|c|c|}
\hline & Precontemplación & Contemplación & Decisión & Mantenimiento & Acción & Recaída \\
\hline Correlación & Precontemplación & 1,000 &, $655^{* *}$ &, $507^{* *}$ &,$- 399^{*}$ &,- 214 &, $587^{* *}$ \\
de Pearson & Contemplación &, $655^{* *}$ & 1,000 &, $769^{* *}$ &,$- 560^{* *}$ &,- 213 &, $714^{* *}$ \\
& Decisión &, $507^{*}$ &, $769^{* *}$ & 1,000 &,- 324 &, 038 &, $470^{* *}$ \\
& Mantenimiento &,$- 399^{*}$ &,$- 560^{* *}$ &,- 324 & 1,000 &, $643^{* *}$ &,$- 630^{* *}$ \\
& Acción &,- 214 &,- 213 &, 038 &, $643^{* *}$ & 1,000 &,- 276 \\
& Recaída &, $587^{*}$ &, $714^{* *}$ &, $470^{* *}$ &,$- 630^{* *}$ &,- 276 & 1,000 \\
\hline
\end{tabular}

* * La correlación es significativa al nivel 0,01 (bilateral); * La correlación es significante al nivel 0,05 (bilateral).

Tabla 4.Correlación entre las subescalas al inicio y seis meses después.

Correlaciones de muestras relacionadas

\begin{tabular}{|l|l|c|c|c|}
\hline \multicolumn{2}{|l|}{} & N & Correlación & Sig. \\
\hline Par 1 & Precontemplación 1 y Precontemplación & 31 &, 786 &, 000 \\
Par 2 & Contemplación 1 y Contemplación & 31 &, 496 &, 005 \\
Par 3 & Decisión 1 y Decisión & 31 &, 558 &, 001 \\
Par 4 & Acción 1 y Acción & 31 &, 589 &, 000 \\
Par 5 & Mantenimiento 1 y Mantenimiento & 31 &, 585 &, 001 \\
Par 6 & Recaída 1 y Recaída & 31 &, 414 &, 020 \\
\hline
\end{tabular}

Tabla 5. Correlación entre las subescalas del ACTA y los cuestionarios que evalúan psicopatología alimentaria.

\begin{tabular}{|lcccccc|}
\hline & Precontemplación & Contemplación & Decisión & \multicolumn{2}{c|}{ Acción } & \multicolumn{2}{c|}{ Mantenimiento Recaída } \\
\hline BSO &, $541^{* *}$ &, $656^{* *}$ &, $667^{* *}$ &,- 229 &,$- 545^{* *}$ &, $574^{* *}$ \\
EAT_40 &, $472^{* *}$ &, $539^{* *}$ &, $492^{* *}$ &,$- 503^{* *}$ &,$- 763^{* *}$ &, $435^{*}$ \\
SINT_BITE &, 078 &, $507^{* *}$ &, $386^{*}$ &,- 110 &,$- 408^{*}$ &, $433^{*}$ \\
SUMA_BITE &, 162 &, $561^{* *}$ &, $443^{* *}$ &,- 099 &,$- 487^{* *}$ &, $488^{* *}$ \\
GRAV_BITE &, 258 &, $544^{* *}$ &, $451^{* *}$ &,- 066 &,$- 520^{* *}$ &, $485^{* *}$ \\
\hline
\end{tabular}




\section{Variables predictoras de psicopatología alimentaria.}

Al introducir en un modelo de regresión múltiple las puntuaciones obtenidas en las diferentes subescalas del cuestionario que mide actitudes ante el cambio, ajustando la edad, se vio que ninguna de ellas era predictora del grado de psicopatología alimentaria medido con el EAT-40 seis meses después. Únicamente las puntuaciones en la fase de mantenimiento resultaron ser un factor protector (coeficiente estandarizado $\beta=-0.665, F=17.454, p<0.001$ ), es decir, aquellas pacientes que presentaban puntuaciones más altas en la fase de mantenimiento, tenían mayor probabilidad de presentar puntuaciones menores en el EAT-40 seis meses después. Este modelo tenía una capacidad de predicción del $66.5 \%$.

Por otra parte, las puntuaciones en la fase de decisión eran predictoras del grado de insatisfacción con la imagen corporal medido con el BSO ( $\beta=0.592$, $t=3.669, F=13.46)$. El modelo tenía una capacidad de predicción del $59.2 \%$. Las puntuaciones en la fase de contemplación predecían las puntuaciones en la subescala de síntomas del BITE seis meses después ( $\beta=0.485, t=2.828, p<0.01)$ con una capacidad de predicción del $40.6 \%$ ( $F=5.12, p<0.05)$

\section{DISCUSIÓN}

La mayoría de las pacientes de nuestro estudio $(45,55 \%)$ se encontraban en fase de acción y, 6 meses después, un $39.4 \%$ continuaba en dicha fase, habiendo aumentado el porcentaje de pacientes en fase de mantenimiento (del 6.4 al 22.4\%). Ello contrasta con otros estudios, tal como el realizado por Treasure et al. (5), en el que la mayoría de los pacientes se encontraban en fase de contemplación. La explicación de estas diferencias reside en que parte de nuestras pacientes habían iniciado tratamiento y, además, el hecho de que los cuestionarios fueran cumplimentados tras una entrevista psiquiátrica, puede tener cierta influencia. En un futuro se pretende llevar a cabo una investigación que inicie la evaluación antes de la primera entrevista y abarque sucesivos momentos de la terapia, ampliando el periodo de estudio, para considerar los diversos factores que puedan estar relacionados con el cambio, así como estudiar las posibles implicaciones pronósticas. En el presente estudio no se encontró correlación significativa entre las puntuaciones en las diferentes fases y variables como el tiempo de evolución del trastorno o tiempo desde el inicio del tratamiento.

Un argumento que habitualmente se esgrime para poner de manifiesto la dificultad de evaluar la actitud frente al cambio para conductas complejas, como son las relacionadas con los trastornos de conducta alimentaria, es que un sujeto puede estar en fase de acción para unos síntomas y en precontemplación para otros, presentando un perfil de puntuaciones altas en varias fases. Nosotros encontramos que las puntuaciones obtenidas en las fases de precontemplación, contemplación, decisión y recaída se correlacionaban entre sí. Por el contrario, las fases de mantenimiento y acción se correlacionan positivamente entre sí e inversamente con precontemplación y recaída. Ello demuestra que el cuestionario está elaborado con cierta validez lógica.

Por otra parte, el hecho de las puntuaciones en las fases de contemplación, decisión y recaída se correlacionen significativamente con las puntuaciones en el EAT y BITE, para detección de conductas anoréxicas y bulímicas respectivamente, habla a favor de que el cuestionario presenta una adecuada validez convergente. Las puntuaciones en las fases de acción y mantenimiento se relacionen inversamente con el EAT-40 y BITE, es decir, a mayores puntuaciones en dichas fases, menor psicopatología alimentaria.

En cuanto a la capacidad de predicción del cuestionario que mide actitudes frente al cambio en los trastornos alimentarios (A.C.T.A.), es importante destacar que las puntuaciones altas en la fase de mantenimiento mostraron ser un factor protector para la psicopatología predominantemente anoréxica, evaluada a través del EAT-40 seis meses después. Las puntuaciones en la fase de contemplación predecían el grado de síntomas bulímicos, medidos con el BITE, seis meses después.

Como se ha señalado, el estudio de los factores que condicionan la actitud frente al cambio en los TCA y su posible valor pronóstico cuenta con numerosas dificultades, pues la sintomatología alimentaria es compleja y la actitud frente a ella suele ser ambivalente y heterogénea. No obstante, consideramos que dicho estudio puede aportar rigor e información al enfoque terapéutico basado en potenciar la motivación para el cambio. El presente estudio pretende mostrar la utilidad de un cuestionario diseñado para evaluar las actitudes ante el cambio en pacientes con trastorno de la conducta alimentaria. En nuestra opinión, sería interesante emplearlo en estudios longitudinales que ayuden a superar las limitaciones anteriormente referidas.

\section{REFERENCIAS}

(1) Vitousek, K., Watson, S., \& Wilson G.T. 'Enhancing motivation for change in treatment-resistant eating disorders.' Clinical Psychology Review. 1998. 18: 391320.

(2) Prochaska, J.O. \& DiClemente, C.C. 'The transtheoretical approach.' In: J.C. Norcross \& I.L. Goldfield (Eds.). Handbook of psychotherapy integration(pp: 300-334). New York: Basic Books. 
(3) Ward, A., Troop, N., Todd, G. \& Treasure, J. 'To change or not to change - "How" is the question?' British Journal of Medical Psychology. 1996. 69: 139-146.

(4) Blake, W., Turnbull, S \& Treasure, J. 'Stages and process of change in eating disorders: Implications for therapy. Clinical Psychology and Psychotherapy. 1997. 4: 186191.

(5) Treasure, J.L., Katzman, M., Schimidt, U., Troop, N., Todd, \& de Silva, P. 'Engagement and outcome therapy in the treatment of bulimia nervosa: First phase of a sequential design comparing motivation enhancement therapy and cognitive behavioural therapy.' Behaviour Research and Therapy. 1999. 37: 405-418.

(6) Prochaska, J.O., DiClemente, C.C. and Norcross, J.C. 'In search of how people change: Applications to addictive behaviors.' American Psychologist, 1992. 47: 1102-1114.

(7) Castro J, Toro J, Salamero M, Guimerá E. 'The Eating Attitudes Test: Validation of the Spanish Version.' Psychological Assessment. 1991. Vol 7, n:2, pp:175-90.

(8) Raich RM, Deus J, Muñoz MJ, Pérez O, Requena A. 'Estudio de actitudes alimentarias en una muestra de adolescentes.' Revista de Psiquiatría de la Facultad de Medicina de Barcelona. 1991. 18: 305-15. 


\section{boletín de suscripción:}

\section{DATOS PERSONALES:}

Nombre y apellidos

NIF

Dirección

Tel.

Población

D.P.

$\mathrm{N}^{\circ}$

Piso

SUSCRIBANME A: "Adicciones". Año 2003

\begin{tabular}{llr} 
España & 4 ejemplares y suplementos & $36,00 €$ \\
& 4 ejemplares ". & $84,00 €$ \\
& 1 ejemplar & $9,00 €$ \\
\multirow{2}{*}{ Extranjero } & 1 monográfico & $13 €$ \\
& 4 ejemplares y suplementos & $55 €$ \\
& 4 ejemplares " & $123 €$ \\
& 1 ejemplar & $14 €$
\end{tabular}

suscripción particular suscripción instituciones

suscripción particular suscripción instituciones

Las suscripciones se entenderán por los cuatro ejemplares del año natural en que se realice la suscripción, sea cual sea el momento del año en que ésta se efectúe.

\section{PAGARÉ:}

A) Por domiciliación bancaria (rellenar para ello la orden de pago que está a continuación).

B) Mediante cheque $n^{\circ}$. que adjunto a nombre de «Adicciones».

C) Transferencia bancaria a LA CAIXA, C/ Olmos, 51 - Palma de Mallorca c/c. 2100.0207.50.0200221610 (Es importante que en la orden de transferencia conste claramente el ordenante de la transferencia para poderla identificar adecuadamente).

$$
\text { .... de }
$$
de 200

(Firma)

\section{ORDEN DE PACO POR DOMICILIACION BANCARIAH}

Nombre del titular de la cuenta

Nombre del Banco o Caja de Ahorros

Número Cuenta Corriente o Libreta (ATENCIÓN: DEBE CONSTAR DE 20 DÍGITOS)

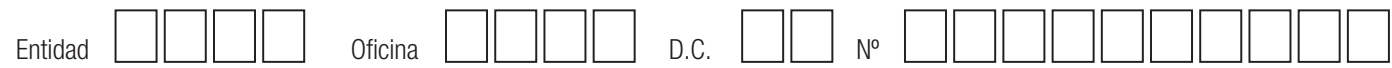

Dirección Banco o C.A.

Calle o Pza.:

Código Postal población

Provincia

Ruego a Vds. Se sirvan tomar nota de que, hasta nuevo aviso, deberán adedudar en mi cuenta los efectos que les sean presentados para su cobro por "Adicciones, Socidrogalcohol"

$$
\text { de }
$$
de 200

Atentamente (firma del titular) 
